\title{
Educar en valores a través del teatro: Un microteatro solidario
}

\author{
Teaching values through theatre: A solidary micro-theatre
}

\author{
Alfredo Blanco Martínez*, Carlos Balado Sáez de Viteri** \\ *Universidade da Coruña, **I.E.S David Buján (Cambre, A Coruña)
}

\begin{abstract}
Resumen
El presente trabajo analiza y describe el proyecto de un docente que utiliza el teatro como recurso para educar en valores a estudiantes de un centro de Educación Secundaria Obligatoria de la provincia de A Coruña. Este estudio se inscribe en el marco de la investigación cualitativa $\mathrm{y}$, más concretamente, emplea el estudio de caso como estrategia metodológica de investigación. Las técnicas para la recogida de datos fueron la entrevista y el análisis de documentos. En conclusión, subrayamos la necesidad de incorporar el teatro al contexto educativo por su carácter pedagógico y fuerza social. Palabras clave: Microteatro, educar, investigación cualitativa, estudio de caso.
\end{abstract}

\begin{abstract}
The present paperwork analyses and describes the project of a teacher that uses theatre as a resource for teaching in values to the students from a Secondary school of A Coruña. This study fits within the framework of qualitative research and, specifically, it uses the case study as a methodological research. The techniques to gather information are the interview and the analysis of documents. At last, we emphasise the necessity to incorporate theatre to the educative context, because it contends a pedagogic role and social strength.

Keywords: Micro-theatre, teaching, qualitative research, case study.
\end{abstract}

\section{Introducción}

Los temas transversales y la educación en valores son la base de todo proyecto educativo. La L.O.E (Ley Orgánica de Educación), en su artículo 121, recoge que "el proyecto educativo del centro recogerá los valores, los objetivos y las prioridades de actuación, así como el tratamiento transversal en las áreas, materias o módulos de la educación en valores y otras enseñanzas".

Los temas transversales son contenidos que forman parte de una educación integral del alumnado. Por ello, desde las distintas áreas curriculares se debe apostar por la educación para la paz, para la salud, para la igualdad, la educación sexual y la educación del consumidor, entre otros.

Una forma de tratar temas y/o problemas que resulten de interés a los estudiantes, es a través del teatro. Se trata de una herramienta de carácter pedagógico con multiplicidad de posibilidades para trabajar en los centros educativos.

De acuerdo con Onieva López (2011); Pérez Fernández (2011) y Cutillas (2015), el teatro es un recurso que persigue la formación humana, académica, lúdica y profesional de los estudiantes. Un formato dramático que resulta muy adecuado y enriquecedor para la labor educativa, es el microteatro.

Tal y como definen Almela; García Lorenzo; Guzmán y Sanfilippo (2013), estas obras se caracterizan por su brevedad, su simplicidad escénica y por su continuidad y simultaneidad. Además, en línea con Orozco Vera (2006) son piezas muy cercanas al público. Por este motivo, entendemos que se trata de un subgénero idóneo para atraer a los estudiantes y para implicarlos en una actividad curricular.

En consecuencia, teatro y educación en valores son dos variables que convergen en el mismo punto, ya que ambas persiguen educar, socializar, concienciar y formar a los individuos en conocimientos científicos y humanos.

Concretamente, el proyecto "microteatro solidario" es una iniciativa planteada por el I.E.S David Buján de Cambre (A Coruña) durante el curso académico 2014/2015. La realización de dicho proyecto estaba prevista para el mes de marzo, concentrando su aplicación en los días 23-27 del presente mes, para conmemorar el día internacional del teatro. Sin embargo, debido a una serie de causas externas, que explicamos en el análisis del trabajo, no pudo llevarse a la práctica quedando reservado para el próximo curso.

El principal objetivo de esta actividad es recoger alimentos para el banco municipal. Para ello, durante una semana, se representan en el Instituto una serie de obras dramáticas en formato de microteatro durante el recreo. Para asistir, el alumnado deberá entregar un quilo de alimentos a cambio de una entrada. Paralelamente, este proyecto pretende involucrar a distintos agentes sociales y económicos del concejo de Cambre para su implicación en la actividad.

El éxito del proyecto, no solo reside en la recogida de alimentos, sino también en el fomento de cooperación entre el alumnado y en su participación en una actividad solidaria.

Los objetivos didácticos que persigue el proyecto son los siguientes:

- Implicar al alumnado en iniciativas solidarias.

- Estimular el sentido de cooperación del alumnado.

- Desenvolver la capacidad creativa del alumnado. 
- Presentar al alumnado y al resto de la sociedad las posibilidades de este subgénero dramático.

- Fomentar la implicación del profesorado en un proyecto solidario.

- Aumentar los lazos de unión entre toda la comunidad educativa del I.E.S David Buján.

Por otra parte, cabe referir que se trata de una actividad que persigue la colaboración y participación activa de todos los miembros de la comunidad educativa. En un primer nivel, contribuye la dirección y los distintos departamentos didácticos. Seguidamente, se encuentra el alumnado que colabora en la organización y control de las obras, así como en la difusión publicitaria a través de la realización de carteles y la utilización de las redes sociales.

En un tercer nivel, se implica el profesorado gestionando y colaborando en las representaciones. En último lugar, el personal administrativo ejerce como apoyo para el montaje de las obras, el control del alumnado, la preparación de los lugares de representación y en la limpieza de las zonas utilizadas.

En suma, este proyecto pretende destinar una semana del curso escolar al microteatro solidario para acercar al alumnado al género teatral y a este subgénero en particular y, al mismo tiempo, para fomentar valores cooperativos y solidarios.

\section{Método}

De acuerdo con Flick (2015), la investigación cualitativa nos ha permitido conocer, comprender $y$ describir la propuesta de trabajo de un docente de Secundaria y, al mismo tiempo, nos ha posibilitado el acceso a documentos y materiales que enriquecen la experiencia del profesional. Por esta razón, hemos empleado el estudio de caso como estrategia metodológica de investigación. En línea con Simons (2011), se trata de un estudio de caso único, ya que nos centramos en único docente con unas determinadas características.

Este trabajo tiene la finalidad de analizar y describir el proyecto "Microteatro solidario" para identificar tanto los beneficios y dificultades que derivan del mismo. Paralelamente, contemplamos los siguientes objetivos específicos:

- Descubrir la perspectiva de un docente al servicio de la innovación y de la mejora educativa.

- Explicar las pautas de un proyecto al servicio de la tarea educativa.

- Conocer la finalidad educativa del proyecto.

\section{Participante}

En este estudio ha colaborado un docente con una dilatada trayectoria en el ámbito de la enseñanza secundaria. Se trata de un profesor de Lengua Castellana y Literatura con más de 20 años de experiencia en un centro público de la Comunidad Autónoma de Galicia.

Asimismo, cabe señalar que el investigado se caracteriza por tener interés por el cambio y mejora educativa a través de la innovación. Entre sus diversas actividades educativas, sobresalen aquellas vinculadas al teatro como recurso educativo.

\section{Instrumentos}

Asumiendo las afirmaciones de Alonso (2007) y Kvale (2011), la entrevista es una técnica que activa la posibilidad de diálogo entre el entrevistador y el entrevistado y que, del mismo modo, permite la recogida de información sobre un tema y/o asunto de interés desde la perspectiva del investigado.

En este caso, hemos utilizado la entrevista de tipo cualitativo, también denominada entrevista en profundidad. Teniendo en cuenta las consideraciones de Taylor y Bodgan (1986), este tipo de entrevista nos ha facultado para conocer de cerca la experiencia, las ideas, los pensamientos y las acciones de un docente de Secundaria.

En suma, la entrevista llevada a cabo con el participante ha sido la siguiente:

- Entrevista "Proyecto Educativo" (EP), con un total de 30 preguntas, orientadas a conocer la perspectiva del docente acerca del "Microteatro solidario".

También, hemos de referirnos al análisis de documentos como técnica para la recogida de datos. Siguiendo a autores como Simons (2011) y Rapley (2014), entendemos que dicho análisis favorece la comprensión del objeto de estudio. Concretamente, hemos examinado el proyecto del docente para descubrir los objetivos y finalidades didácticas, así como la metodología y los recursos necesarios para ponerlo en marcha.

\section{Procedimiento}

Tras la recogida de los datos, debemos proceder con el respectivo análisis. En consonancia con Bodgan y Biklen (1982) y Gibbs (2012), esta tarea nos descubre el conocimiento científico generado $\mathrm{y}$, al mismo tiempo, el que queremos transmitir.

Para el análisis de los datos, hemos tomado como referente el esquema de Miles y Huberman (1994). Estos autores proponen un análisis centrado en los siguientes procesos: reducción, exposición, conclusión y verificación de los datos.

De este modo y teniendo en consideración el análisis DAFO, planteamos el siguiente proceso de codificación y análisis de los datos. Véase la Figural:

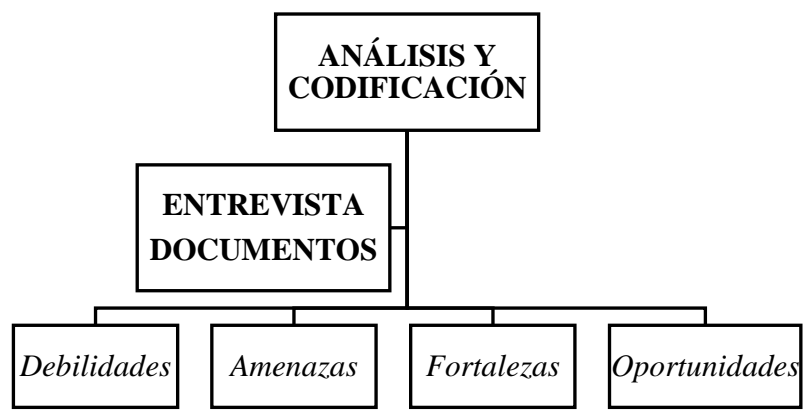

Figura 1. Esquema de codificación y análisis de los datos. 


\section{Resultados}

La información que presentamos se organiza en torno a las dimensiones del esquema de análisis DAFO. Hemos considerado muy oportuno dicho esquema, ya que el proyecto no pudo ponerse en marcha durante el curso académico 2014/2015.

\section{Debilidades}

En el presente apartado, trataremos de abordar aquellas flaquezas que han repercutido en el proyecto. Por un lado, el docente nos comenta que la colaboración de las compañías teatrales es totalmente altruista y esto provoca "que si les surge un trabajo remunerado, abandonen su participación desinteresada" (EP, p. 3). Por este motivo, nos explica que "la falta de profesionalidad de mucha gente dedicada al teatro, hace que sea gente que rompe con facilidad sus compromisos" (EP, p. 3).

Por otra parte, el entrevistado apuesta por solventar dichas debilidades en un futuro próximo. En este sentido, afirma que la solución es explicar a los grupos de teatro "el proyecto con detenimiento y mentalizándolos de la importancia de mantener el compromiso de trabajo para, así, concienciarlos de los prejuicios que pueden ocasionar su falta de asistencia” (EP, p. 4).

De este modo, comprendemos que la principal debilidad reside en el llamamiento de los grupos teatrales. No obstante, a través de la toma de concienciación y el establecimiento de un acuerdo previo, se puede llevar a cabo el proyecto en los centros con éxito.

\section{Amenazas}

Por amenazas entendemos aquellos peligros que han repercutido en la puesta en marcha del proyecto. Nuevamente, el profesor nos comenta que "la poca seriedad de las personas que se comprometen a traer sus obras de forma desinteresada a los Institutos, es la principal amenaza" (EP, p. 5). Por ello, nos explica que los riesgos de este calibre, provocan que "los proyectos de esta índole se vengan abajo" (EP, p. 6).

Con visión de mejora, el entrevistado nos aclara que "a pesar de que sea un trabajo duro y continuo, es vital poner en marcha la primera edición para conseguir publicidad y repercusión social $\mathrm{y}$, de esta manera, provocar la atención de especialistas en teatro" (EP, p. 7).

Por tanto, teniendo en cuenta las aportaciones del participante, consideramos que la óptima organización y distribución del tiempo, permitirá destruir toda amenaza externa.

\section{Fortalezas}

A continuación, atenderemos a aquellas fuerzas positivas que rodean al proyecto educativo. El análisis del documento y del testimonio del investigado, son muestra de la implicación de toda la comunidad educativa. En relación con esto, el profesor nos aclara que "este tipo de trabajo permite que todos trabajemos en equipo, desde el diseño del cartel hasta la planificación y la preparación de los escenarios" (EP, p. 7). Por esta razón, entendemos que el proyecto de teatro tiene en su haber una fuerza que hace imprescindible seguir trabajando para sacarlo adelante.
Paralelamente, pensamos que este plan educativo condensa toda una serie de elementos que hacen que sea íntegro desde cualquier perspectiva, ya que, en un espacio de tiempo bastante corto, concentra temas variados y transversales, así como el desarrollo de las competencias básicas de los estudiantes.

\section{Oportunidades}

Este trabajo es una ocasión para trabajar en valores con el alumnado de los centros. Además, es una actividad que tiene una repercusión inmediata en los estudiantes porque capta su atención y provoca que valoren la labor docente. En este sentido, el entrevistado nos explica que "el microteatro permite poner en marcha una reflexión a través de distinto temas con una finalidad solidaria" (EP, p. 2).

También, cabe señalar que el microteatro solidario posibilita que la comunidad educativa de entrada a los siguientes temas transversales:

- Conocer la realidad social más cercana.

- La empatía con el diferente.

- La colaboración como una metodología de trabajo.

- La iniciativa individual.

- Potenciar la autoestima del alumnado.

- La competencia artística.

- La importancia social de una buena comunicación verbal y paraverbal.

En suma, deducimos que la principal coyuntura del proyecto es otorgar la importancia que se merece a la transversalidad y al teatro como herramienta pedagógica. Así, el centro se convierte en un espacio para el diálogo, el debate y la discusión desde el respeto y la tolerancia.

\section{Discusión}

En este trabajo, la utilización de una metodología cualitativa y, más concretamente, del estudio de casos como estrategia metodológica de investigación, nos ha posibilitado comprender y reflexionar sobre la acción de un docente que persigue la transformación y mejora de la educación. Asimismo, la realización de dicha investigación nos ha proporcionado conocimiento sobre proyectos que emplean el teatro como recurso educativo y que integran a toda la comunidad educativa.

En relación con la fundamentación teórica y con los objetivos formulados al principio, así como con los resultados obtenidos en el estudio empírico, podemos presentar las siguientes conclusiones:

En primer lugar, el testimonio del investigado ha puesto de manifiesto las ventajas y desventajas de llevar a cabo un proyecto solidario en un centro educativo. Teniendo en cuenta las características del mismo, entendemos que las mayores dificultades residen en los factores externos, como es contactar con las compañías de teatro y sellar un compromiso, y en la circunstancia temporal, ya que una inadecuada temporalización puede desterrar este tipo de trabajo. No obstante, el hecho de implicar a toda la comunidad educativa $\mathrm{y}$, al mismo tiempo, propiciar una educación integral a través de 
principios solidarios y cooperativos, permite superar todo obstáculo.

Paralelamente, la voz del docente nos ha descubierto iniciativas, como el proyecto "microteatro solidario", que se están organizando en los centros de Secundaria para proporcionar y dotar al alumnado de valores formativos, ciudadanos y democráticos. En este sentido, cabe subrayar el lugar que ocupa el teatro como nexo de unión entre el elemento curricular y el lúdico.

Todo proyecto de centro incluye unos objetivos, unos contenidos, una metodología, unos criterios y/o procedimientos. $\mathrm{Al}$ ser un proyecto que por su ambición abarca a la mayoría de la comunidad educativa, es necesario presentarlo en el concejo escolar, al claustro de profesores y contar con el apoyo de la dirección. El análisis del documento, nos ha revelado la importancia de implicar a toda la comunidad educativa, porque su fortaleza es la que faculta este tipo de planes educativos.

En último lugar y en estrecha relación con el punto anterior, cabe referirse a la finalidad educativa del proyecto. La realización de este estudio, nos ha mostrado la importancia de transformar los centros en escenarios teatrales, sin alterar de forma significativa el funcionamiento de la actividad académica, para fomentar entre el alumnado valores de cooperación y solidaridad. Por esta razón, comprendemos que esta experiencia busca que los estudiantes sean más solidarios, con mayor empatía, con un mayor grado de autoestima y más creativos.

De acuerdo con estudios recientes sobre el teatro como las investigaciones de Motos (2009); Blanco Martínez y González Sanmamed (2014), consideramos el teatro un recurso pedagógico cualificado, por un lado, para crear un ambiente de enseñanza y aprendizaje y, por otra parte, para que los estudiantes ejerzan la ciudadanía activa y desarrollen sus habilidades sociales. Además, al tratarse de un proyecto de centro, comprendemos que el teatro se convierte en la pieza angular para el ejercicio comunicativo y el diálogo.

En lo que respecta a las limitaciones, podemos comentar que el hecho de no desanimarse durante todo el proceso es fundamental. A pesar de que resulte una tarea ardua contactar y comprometer a las compañías de teatro, hay que persistir en la idea de un trabajo continuo. En este sentido, también es interesante buscar alternativas en el propio centro, como que los alumnos representen las obras, para evitar todo condicionamiento exterior.

Como investigaciones futuras, sería interesante realizar un análisis comparativo con otros proyectos de centro que contemplen el teatro como recurso para una educación en valores, para observar las similitudes y los beneficios, así como para aprender e intercambiar experiencias.

En definitiva, asumiendo las afirmaciones de Rodríguez López-Vázquez (2008) y García del Toro (2011), entendemos que el teatro es un recurso que se ofrece a la enseñanza como vehículo conductor de cualquier tema y con una gran flexibilidad de método.

\section{Referencias}

Alonso, L. E. (2007). Sujetos y Discurso: el lugar de la entrevista abierta en las prácticas de la sociología cualitativa. En J. M. Delgado \& J. M Gutiérrez (Ed). Métodos y técnicas cualitativas de investigación en Ciencias Sociales (pp. 225-240). España: Síntesis.

Almela, M., Lorenzo Ma. G., Guzmán, H. \& Sanfilippo M. (2013). Mujeres a la conquista de espacios. Madrid: UNED.

Blanco Martínez, A. y González Sanmamed, M. (2014). Acercar el microteatro al aula. Una experiencia con alumnado de educación secundaria. Revista Internacional Digilec, 1, 94-114.

http://digilec.udc.es/files/ASTROLABIO1_y61azv00. pdf

Bodgan, R. \& Biklen, S. (1982). Qualitative research for education: an introduction to theory and methods. Boston: Allyn y Bacon.

Cutillas Sánchez, V. (2015). El teatro y la pedagogía en la historia de la educación. Tonos Digital, 28, 1-31. http://www.tonosdigital.es/ojs/index.php/tonos/article/ view/1215/745

Flick, U. (2015). El diseño de la investigación cualitativa. Madrid: Morata.

García del Toro, A. (2011). Teatralidad. Cómo y por qué enseñar textos dramáticos. Barcelona: Graó.

Gibbs, G. (2012). El análisis de datos cualitativos en investigación cualitativa. Madrid: Morata.

Kvale, S. (2011). Las entrevistas en investigación cualitativa. Madrid: Morata.

Miles M.B. \& Huberman A.M. (1994). Qualitative Data Analysis: An expanded sourcebook. Thousand Oaks, California: SAGE.

Motos, T. (2009). El teatro en la educación secundaria: fundamentos y retos. Creatividad y sociedad, 29, 1-35. http://centroderecursos.alboan.org/ebooks/0000/0847/ 5 APY REE 2.pdf

Onieva López, J. L. (2011). La dramatización como recurso educativo: estudio comparativo de una experiencia con estudiantes malagueños de un centro escolar concertado y adolescentes puertorriqueños en situación de marginalidad. Tesis doctoral, Departamento de Lengua y Literatura, Universidad de Málaga, Málaga, España.

http://riuma.uma.es/xmlui/bitstream/handle/10630/48 92/TDR\%20ONIEVA\%20LOPEZ.pdf? sequence=1

Orozco Vera, Ma . J. (2006). El teatro breve español de las últimas décadas: tradición y vanguardia. Cuadernos del Ateneo, 21, 29-34.

http://dialnet.unirioja.es/servlet/articulo? codigo $=2360$ 569

Pérez Fernández, J. (2011). Motivar en secundaria. El teatro: una herramienta eficaz. Barcelona: Ediciones Erasmus.

Rapley, T. (2014). Los análisis de conversación, de discurso y de documentos en investigación cualitativa. Madrid: Morata. 
Rodríguez López-Vázquez, A. (2008). Aprender a ver teatro. Empezar a hacer teatro. Revista de Literatura-Especial teatro, 233, 75-81.

Taylor, S.J. \& Bodgan, R. (1986). Introducción a los métodos cualitativos de investigación. Buenos Aires: Paidós.

Simons, H. (2011). El estudio de caso. Teoría y práctica. Madrid: Morata.

\section{Agradecimientos}

Con especial gratitud al I.E.S David Buján de Cambre (A Coruña) y a toda la comunidad educativa que lo integra, por hacer de la innovación y del teatro un camino para el cambio y la mejora educativa. 